\title{
Fast Vortex Core Switching at Moderate Temperatures
}

\author{
K.M. LEBECKI ${ }^{a, *}$ AND U. NOWAK \\ ${ }^{a}$ Nanotechnology Centre, VSB-Technical University of Ostrava, 17. listopadu 15, CZ-708 33 Ostrava, Czech Republic \\ ${ }^{b}$ Department of Physics, University of Konstanz, D-78457 Konstanz, Germany

\begin{abstract}
Ferromagnetic vortex core switching is investigated using micromagnetic simulations. For that the OOMMF program is used together with a temperature extension we have developed recently. This is a continuum micromagnetic approach, where the well-known Landau-Lifshitz-Gilbert equation (valid for zero temperature) is replaced by the Landau-Lifshitz-Bloch equation. In our research we simulate switching of a ferromagnetic vortex core in a flat disk (diameter $200 \mathrm{~nm}$, thickness $20 \mathrm{~nm}$ ) with material parameters that resemble permalloy. Temperatures in the range $400 \mathrm{~K}$ to $700 \mathrm{~K}$ are considered. Switching itself is caused by application of a very short oscillating magnetic pulse. Parameters used resemble conditions met in the experiment: oscillation period 141 ps (equal to the peak width) and amplitude $60 \mathrm{mT}$. Surprisingly, no large temperature- or discretization dependence is found. Reasons for that are discussed.
\end{abstract}

DOI: 10.12693 /APhysPolA.127.342

PACS: 75.30.Ds, 75.60.Nt, 75.78.-n, 75.78.Cd

\section{Introduction}

A ferromagnetic vortex is an in-plane domain structure with a rotational character except of its very center - called core - where the magnetization points out-ofplane [1]. This magnetic texture has attracted quite a lot of interest recently, possible applications in magnetic memory devices [2] or GHz-frequency generators [3] have been proposed. Vortex core is very thin, usually with a few $\mathrm{nm}$ in diameter. Vortex core switching (VCS) can happen either in a quasi-static way - e.g. by applying an orthogonal, slowly increasing magnetic field [4, 5], or dynamically - e.g. by applying magnetic or electric pulses $[6,7]$, or by applying oscillating magnetic or electric fields $[2,3,8-10]$. In every case the VCS is accompanied by a short presence of a very unstable object - the Bloch point (BP) [6], see Fig. 1. The BP is

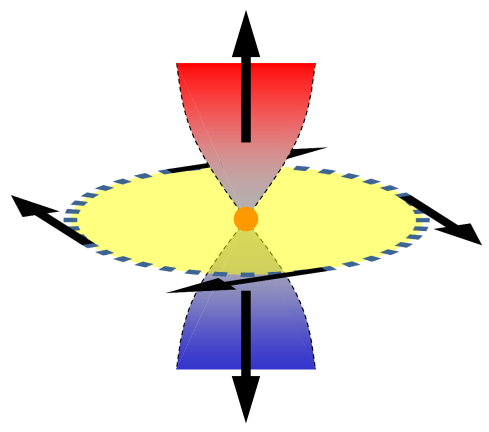

Fig. 1. Schema of a Bloch point - a very unstable object mediating the vortex core reversal. Arrows indicate the magnetization direction close to the Bloch point, placed in the center (orange-marked). Colors are used to stress (i) the vertical tendency close to the vertical axis (red-blue) (ii) the rotational tendency close to the horizontal plane containing the Bloch point (dark yellow).

*corresponding author; e-mail: lebecki@fuw.edu.pl a mathematical singularity, thus proper description of the VCS is very difficult $[4,5]$. Only at an elevated temperature this problem can be avoided [5, 11]. Thus, we present here results of our latest study, where fast VCS in a wide temperature range is simulated.

\section{Methods}

We follow a temperature-dependent micromagnetic approach, based on the Landau-Lifshitz-Bloch equation of motion [12]. This equation is an extension of the well-known Landau-Lifshitz-Gilbert (LLG) equation of motion for the case of non-zero temperature, $T \geq 0$. It can be written in the form [12]:

$$
\begin{gathered}
\dot{\boldsymbol{M}}=-\bar{\gamma} \boldsymbol{M} \times H_{\mathrm{eff}}+\bar{\gamma} \alpha_{\|} \frac{M_{\mathrm{s}}}{M^{2}}\left(\boldsymbol{M} \cdot H_{\mathrm{eff}}\right) \boldsymbol{M} \\
+\bar{\gamma} \alpha_{\perp} \frac{M_{\mathrm{s}}}{M^{2}}\left(\boldsymbol{M} \times H_{\mathrm{eff}}\right) \times \boldsymbol{M}, \\
\alpha_{\|}=\alpha 2 T / 3 T_{\mathrm{C}}, \quad \alpha_{\perp}=\alpha\left(1-T / 3 T_{\mathrm{C}}\right) .
\end{gathered}
$$

Here, $\bar{\gamma}$ is the gyromagnetic ratio, $M$ is the magnetization, $H_{\text {eff }}$ is the effective field, $M_{\mathrm{s}}$ is the saturation magnetization at $T=0, M=|\boldsymbol{M}|, \alpha$ is the Gilbert damping constant at the atomic level, $T_{\mathrm{C}}$ is the Curie temperature, and only the case $T \leq T_{\mathrm{C}}$ is considered. The effective field is similar to the case of the LLG equation,

$$
H_{\mathrm{eff}}=\boldsymbol{H}_{\mathrm{d}}+\boldsymbol{H}_{\mathrm{ext}}+\frac{2 A \nabla^{2} \boldsymbol{M}}{\mu_{0} M_{\mathrm{e}}^{2}}-\left(\frac{M^{2}}{M_{\mathrm{e}}^{2}}-1\right) \frac{\boldsymbol{M}}{2 \chi_{\|}},
$$

where $\boldsymbol{H}_{\mathrm{d}}$ and $\boldsymbol{H}_{\text {ext }}$ are, respectively, the demagnetization- and the external field, $A$ is the exchange constant, $\mu_{0}$ is the vacuum permeability, $M_{\mathrm{e}}$ describes (temperature dependent) equilibrium magnetization length, and $\chi_{\|}$is the longitudinal susceptibility [12].

In our research we have simulated a flat disk with material parameters resembling permalloy [5, 11, 13]. Its diameter was $200 \mathrm{~nm}$, its thickness was $20 \mathrm{~nm}$. Fast VCS was achieved by applying a very short (pulseshaped [14]), rotating in-plane magnetic field, see Fig. 2a. 

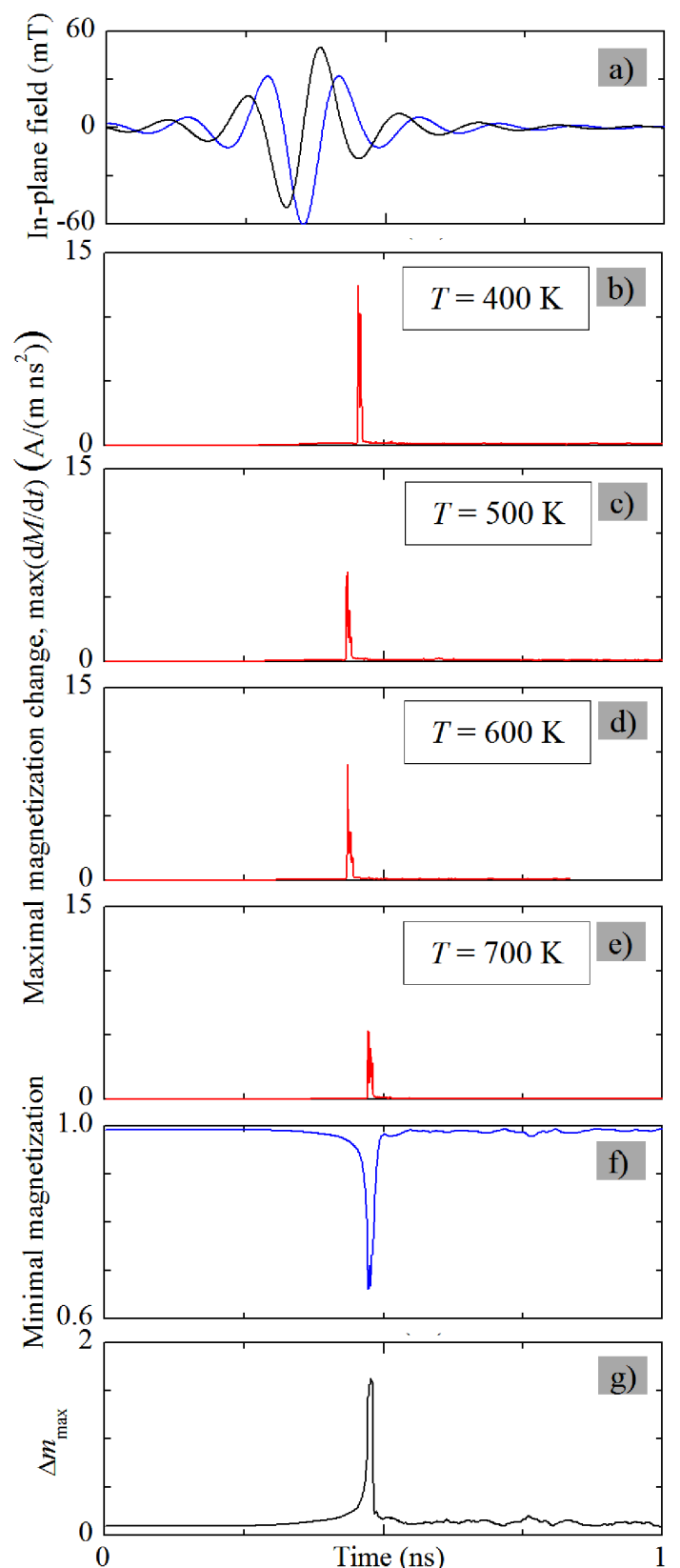

Fig. 2. Applied in-plane magnetic field, pulseshaped (a). We show here both in-plane field components. Maximum of the magnetization change, $\max (\mathrm{d} M / \mathrm{d} t)$, for the indicated temperatures (b)-(e). Alternative parameters associated with the switching: minimum magnetization length in the sample (f) and maximal magnetization change between neighboring cells $(\mathrm{g})$ - both normalized; in both cases $T=700 \mathrm{~K}$. In all cases discretization grid no. 4 was used.

Thus, sub-100 ps switching was achieved, in accordance with earlier studies $[9,10]$. The pulse shape was carefully chosen to mimic the experimentally available conditions, cf. Fig. $2 b$ in Ref. [10]. Such a switching is caused by an excitation of spin waves. Following notation introduced in Ref. [9] we have excited the azimuthal spin wave with radial mode number $n=1$ and $\mathrm{CW}$ sense of rotation, $m=-1$; initial vortex state had CCW chirality and a positive polarity, $p=1$. According to the experiment, it is easier to switch the vortex with a $\mathrm{CW}$ excitation, in such a case the excitation field is smaller [9]. This was also our case - we used a CW excitation with a period 141 ps. We point out that the peak amplitude we had to use $(60 \mathrm{mT})$ is larger compared to Ref. [10] because of a smaller sample size considered here. According to the literature using appropriate discretization cell size is a crucial point when simulating BP-related phenomena $[4,5,11,15]$. This is because of the complex nature of this object: when approaching it from opposite directions, the magnetization sign changes, see Fig. 1. Thus, "any direction of the magnetization is present in the close vicinity of a Bloch point" [16]. One has also to account for the small radius of magnetization-squeezing around this point $[5,11]$. All this makes the Bloch point calculations very sensitive on the chosen cell size. Thus, we have performed a series of simulations for different grids listed in Table.

\section{TABLE}

List of used mesh grids. Every cell was a rectangular prism with dimensions $c_{x} \times$ $c_{y} \times c_{z}$. The simulated disk was orthogonal to the $z$-axis.

\begin{tabular}{c|l|l|l}
\hline \hline $\begin{array}{c}\text { Grid } \\
\text { number }\end{array}$ & $c_{x}[\mathrm{~nm}]$ & $c_{y}[\mathrm{~nm}]$ & $c_{z}[\mathrm{~nm}]$ \\
\hline 1 & 0.097656 & 0.097656 & 2.5 \\
2 & 0.195312 & 0.195312 & 0.625 \\
3 & 0.392157 & 0.392157 & 0.392157 \\
4 & 0.645161 & 0.645161 & 0.645161 \\
5 & 0.8 & 0.8 & 0.8 \\
6 & 1 & 1 & 1
\end{tabular}

\section{Results}

Results presented in Fig. 2 are representative for all used grids. Temperature dependence of the switching time can be observed in Fig. 2b-e. Here, the maximum magnetization change across all the cells are plotted, $\max (\mathrm{d} M / \mathrm{d} t)$ - a handy parameter allowing to define the exact moment of the switching $[4,9,10]$. No special temperature dependence can be seen in Fig. 2. Figure 2f, $\mathrm{g}$ shows other possible parameters related to the VCS [5], respectively: minimal magnetization length, normalized, and simulation quality/validity parameter $\Delta m_{\max }$ [11]. The last one is the maximal difference in magnetization for nearby cells (neighbors), normalized. In Ref. [11], where we have introduced this parameter, we also suggested its maximal value, a signature of appropriate micromagnetic simulations: 0.5 . High $\Delta m_{\max }$ values found in our present study exceed remarkably this threshold. This is true for all temperatures and for all grids and it means that our results might not be reliable, despite using a much finer grid as compared to other reports. This is in our opinion the reason why the results in Fig. 2 
have no clear temperature dependence. BP-related simulations seem to be more complex than hitherto expected. One possibility would be to perform simulations at even higher temperatures, where the $\Delta m_{\max }$-problem ought to be smaller [11]. An even better approach was suggested recently by Andreas et al. [15]: to combine and perform in-parallel micromagnetic (continuum) simulations with atomistic (discrete) calculations.

\section{Summary}

We have numerically evaluated fast vortex core switching caused by a short oscillating magnetic field pulse. No remarkable temperature-dependence was found in the investigated region, from $400 \mathrm{~K}$ to $700 \mathrm{~K}$. Also, no special grid-dependence was found, despite going down with the discretization cells up to sub-nanometer values. These strange results need further investigations. One cannot, however, exclude that the nature of the Bloch point is more complex than so far expected. Maybe, the continuum micromagnetic approach should be supported/replaced by a discrete atomistic study. Results shown here present challenges that need to be solved and questions that need to be answered while researching the phenomenon of the vortex core switching. Raised questions are important for further vortex studies.

\section{Acknowledgments}

This work was partially supported by the research Grant No. CZ.1.07/2.3.00/30.0055 of the Operational Programme Education for Competitiveness and cofinanced by the European Social Fund and the state budget of the Czech Republic. This work was also supported by the IT4Innovations Centre of Excellence project (CZ.1.05/1.1.00/02.0070), funded by the European Regional Development Fund and the national budget of the Czech Republic via the Research and Development for Innovations Operational Programme, as well as Czech Ministry of Education, Youth and Sports via the project Large Research, Development and Innovations Infrastructures (LM2011033). Financial support by the Baden-Württemberg Stiftung is gratefully acknowledged. Finally, we thank Dr. Rudolf Sýkora for reading our manuscript.

\section{References}

[1] A. Wachowiak, J. Wiebe, M. Bode, O. Pietzsch, M. Morgenstern, R. Wiesendanger, Science 298, 577 (2002).

[2] K. Nakano, D. Chiba, N. Ohshima, S. Kasai, T. Sato, Y. Nakatani, K. Sekiguchi, K. Kobayashi, T. Ono, Appl. Phys. Lett. 99, 262505 (2011).

[3] Q. Mistral, M. van Kampen, G. Hrkac, J.-V. Kim, T. Devolder, P. Crozat, C. Chappert, L. Lagae, T. Schrefl, Phys. Rev. Lett. 100, 257201 (2008).

[4] A. Thiaville, J.M. Garcia, R. Dittrich, J. Miltat, T. Schrefl, Phys. Rev. B 67, 094410 (2003).

[5] K.M. Lebecki, U. Nowak, Phys. Rev. B 89, 014421 (2014).

[6] R. Hertel, S. Gliga, M. Faehnle, C.M. Schneider, Phys. Rev. Lett. 98, 117201 (2007).

[7] Y. Liu, S. Gliga, R. Hertel, C. Schneider, Appl. Phys. Lett. 91, 112501 (2007).

[8] B. Van Waeyenberge, A. Puzic, H. Stoll, K.W. Chou T. Tyliszczak, R. Hertel, M. Fahnle, H. Bruckl, K. Rott, G. Reiss, I. Neudecker, D. Weiss, C.H. Back, G. Schuetz, Nature 444, 461 (2006).

[9] M. Kammerer, M. Weigand, M. Curcic, M. Noske, M. Sproll, A. Vansteenkiste, B. Van Waeyenberge, H. Stoll, G. Woltersdorf, C.H. Back, G. Schuetz, Nat. Commun. 2, 279 (2011).

[10] M. Kammerer, H. Stoll, M. Noske, M. Sproll, M. Weigand, C. Illg, G. Woltersdorf, M. Fähnle, C. Back, G. Schütz, Phys. Rev. B 86, 134426 (2012).

[11] K.M. Lebecki, D. Hinzke, U. Nowak, O. ChubykaloFesenko, Phys. Rev. B 86, 094409 (2012).

[12] D.A. Garanin, Phys. Rev. B 55, 3050 (1997).

[13] K.M. Lebecki, U. Nowak, J. Appl. Phys. 113, 023906 (2013).

[14] Applied field was constructed from a sinusoid multiplied by a Lorentzian with the full width at half maximum (FWHM) equal to one period.

[15] C. Andreas, S. Gliga, R. Hertel, J. Magn. Magn. Mater. 362, 7 (2014).

[16] R. Hertel, J. Kirschner, J. Magn. Magn. Mater. 278, L291 (2004). 